\title{
Ultra-narrowband Emitters/Absorbers Based on Metal-Insulator-Metal Stacks for Nondispersive Infrared Gas Sensors
}

\author{
Aisheng Yu, ${ }^{1,2}$ Wei Li ${ }^{3}$ Yuelin Wang, ${ }^{1}$ and $\mathrm{Tie}^{\mathrm{Li}}{ }^{1 *}$ \\ ${ }^{1}$ Science and Technology on Micro-System Laboratory, Shanghai Institute of Microsystem \\ and Information Technology, Chinese Academy of Sciences, Shanghai 200050, China \\ ${ }^{2}$ University of Chinese Academy of Sciences, Beijing 100049, China \\ ${ }^{3}$ State Key Laboratory of Functional Materials for Informatics, Shanghai Institute of Microsystem \\ and Information Technology, Chinese Academy of Sciences, Shanghai 200050, China
}

(Received May 23, 2018; accepted August 10, 2018)

Keywords: metamaterial, plasmonics, nondispersive infrared, sensors

Native narrowband emitters/absorbers in the mid-infrared range are highly demanded in nondispersive infrared (NDIR) gas sensors, which not only decrease the complexity of the whole system, but also improve their performance. Metamaterials with narrowband features are considered as high-potential candidate materials for NDIR gas sensors because of their low cost, small volume, and almost arbitrary working band. Recently, narrowband emitters based on metamaterials have been proposed and proved to be feasible; however, the inherent losses in metals limit the ability to achieve ultra-narrowband emitters/absorbers. In order to break through this inherent defect in metamaterials and realize high-performance NDIR gas sensors, surface lattice resonance (SLR) based on parallel coupling between the localized surface plasmon resonance (LSRR) and the lattice diffraction in metamaterials has been obtained both in the simulation and experiment in this work. In the results, the bandwidth of the SLR in the mid-infrared range is an order of magnitude lower than traditional LSRR; besides, the strength of SLR can be modulated by the insulator expediently. The results may fuel the launch of this delocalized parallel coupling to practical applications for realizing ultra-narrowband resonance for high-performance NDIR gas sensors.

\section{Introduction}

Nondispersive infrared (NDIR) sensors, based on the finger-print absorption of gases or liquids in the mid-infrared range, are in high demand owing to increasing number of requirements for indoor air or outdoor portable environment monitoring because of features such as their low cost, miniature sensing systems, and small volume. However, a conventional NDIR system contains an optical filter to tailor the broadband spectrum into a narrowband, which not only wastes most of the energy, but also increases the complexity of the whole system. Driven by this requirement, metal-insulator-metal (MIM) structures based on localized surface

${ }^{*}$ Corresponding author: e-mail: tli@mail.sim.ac.cn https://doi.org/10.18494/SAM.2018.2001 
plasmon resonances (LSPRs) have recently been presented as compact, small volume, and filterfree (low-power dissipation) thermal infrared sources for NDIR gas sensors. ${ }^{(1-3)}$

MIM structures are also known as metamaterial perfect absorbers, which exhibit nearly perfect absorption at specific wavelengths ${ }^{(4-7)}$ by confining the electromagnetic wave from free space to electric dipoles inside resonators. In the mid-infrared range, collective motions of electrons inside microparticles, namely, LSPRs, contribute to the selective absorption or emission. Narrowband applications can be realized using metamaterials with uniform dimensional metallic particles, while broadband applications can be realized using metamaterials with multidimensional metallic particles. ${ }^{(8,9)}$ However, the development of ultra-narrowband applications is limited owing to large ohmic losses inherent in all metals. In addition, surface lattice resonances (SLRs) with ultra-narrowbands are also investigated in metallic particle arrays, ${ }^{(10-14)}$ which have the potential to replace LSPRs or improve the performance of LSPRs in ultra-narrowband applications. Moreover, the near-field enhanced SLR has also been utilized for surface-enhanced Raman scattering ${ }^{(15)}$ or refractive index sensing ${ }^{(16)}$ on Metallic particle array fabricated on a transparent Substrate (MS) structures.

Diffractive coupling between metallic particles is considered to be the origin of this lattice resonance; hence, SLRs can be well predicted near, but not exactly at Rayleigh anomalies (RAs). ${ }^{(17,18)}$ RAs are attributed to the transitional process of the diffraction order transforming from evanescent to radiative, which also indicates the wave number in the vertical direction transforming from an imaginary to a real number. The corresponding $\lambda_{R A}$ under oblique incident light can be calculated as

$$
\begin{aligned}
& \lambda_{R A}^{ \pm 1,0}=a_{x} n_{e}(1 \pm \sin \theta), \\
& \lambda_{R A}^{0, \pm 1}=a_{y} n_{e} \sqrt{1-\sin ^{2} \theta},
\end{aligned}
$$

where $n_{e}$ is the refractive index of the environment, $\theta$ is the angle of incidence, and $a_{x}$ and $a_{y}$ indicate the lattice constants along different axes.

In the above equations, TE-polarized light is adopted and the in-plane components of the incident wave vector propagate along the $x$-axis. $\mathrm{RA}( \pm 1,0)$ and $\mathrm{RA}(0, \pm 1)$ can be explained as diffraction orders with propagation directions along the $x$ - and $y$-axes, respectively. Obviously, SLRs can be classified into parallel couplings [LSPR coupling to RA $(0, \pm 1)$ ] and orthogonal couplings [LSPR coupling to $\mathrm{RA}( \pm 1,0)$ ]. Orthogonal couplings have been widely studied in the MS structure, ${ }^{(19-22)}$ in which the homogenous medium requirement must be satisfied to avoid the suppression of diffraction by the mismatch index around the arrays. Recently, parallel coupling has also been demonstrated to show a stronger vertical field delocalization, ${ }^{(23,24)}$ possibly making it more suitable than orthogonal coupling in applications for sensing or enhancing emissions.

In this work, SLRs based on parallel coupling have been investigated in MIM structures. Unlike in the MS structure, the coupling efficiency dramatically increases when an insulator with a higher index is adopted, even though the homogenous medium condition is not satisfied. 
In the simulations, the full width at half maximum (FWHM) of SLR is an order of magnitude lower than that of the traditional LSPR in MIM structures, which may be adopted to realize ultra-narrowband emitters/absorbers for high-performance NDIR gas sensors.

\section{Simulations and Experiments}

In this work, MIM structures, working in the mid-infrared range, are studied by both finite different time domain (FDTD) numerical simulations and Fourier transform infrared spectrometry (FTIR). The corresponding trilayer MIM structure is schematically shown in Fig. 1, in which the definition of TE-polarized incident light is also given in Fig. 1(a). Structure parameters are defined in Fig. 1(b), in which the red square indicates the unit cell used in the simulations. SEM images on the right-hand side of Fig. 1(b) are the oblique and cross-sectional views of the unit cell in the experiments. The resonant elements made of gold disks are arranged into a simple square lattice. Diffraction orders propagating along the surface, namely, the RAs in this periodic array, couple with the LSPRs inside each disk, forming Fanolike resonances, SLRs.

In the simulations, periodic boundary conditions were used around the unit cell to describe the infinite square lattice. Light with the polarization direction along the $y$-axis was normally incident on the array, which means that $\theta$ defined in Fig. 1(a) equals zero. The material parameters of gold were described as the fitted curves as per Palike's handbook. ${ }^{(25)}$ Insulators were defined as nondispersive materials with $n=1-2$ and $k=0$, in which $n$ is the refractive index and $k$ is the extinction coefficient. Extinction coefficients describe the losses of electro-magnetic (EM) energy in materials, which affect the magnitude of resonance damping. Metals have considerable losses in the infrared range, while losses in insulators, by contrast, are negligible. Finally, the trilayer MIM structures were immersed in the environment with the refractive index $n_{e}=1.2$ to achieve the interesting condition $n_{e}>n_{i}$.

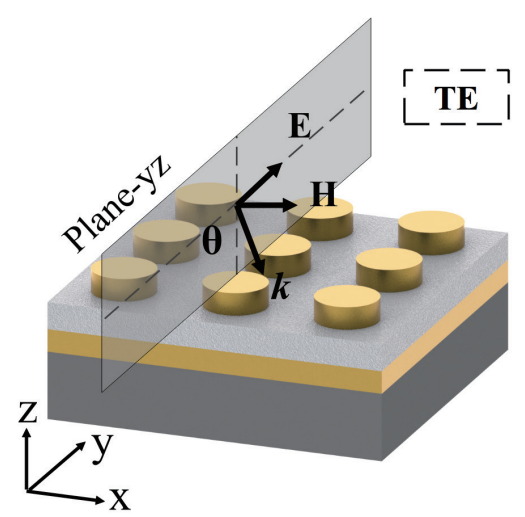

(a)

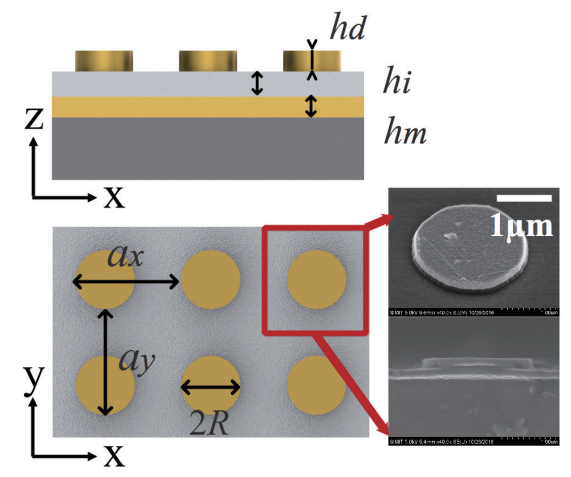

(b)

Fig. 1. (Color online) Schematics of MIM structure in square lattice. Definition of TE-polarized oblique plane wave is given in (a). TM-polarization can be explained as TE-polarization rotated $90^{\circ}$ along the $k$-axis. (b) Definitions of structure parameters: $R$ (radius of disks), $a_{x}$ (grating constant along $x$-axis), $a_{y}$ (grating constant along $y$-axis), $h_{d}$ (thickness of disks), $h_{i}$ (thickness of insulator), and $h_{m}$ (thickness of Au mirror). Fabricated unit cells of MIM are visualized in SEM images at the right of (b) with oblique and cross-sectional views. 
As shown in Fig. 2, SLR $(0, \pm 1)$ near RA $(0, \pm 1)$, marked by the dark arrow, broadens and shows a feeble redshift, while $\operatorname{SLR}( \pm 1,0)$ near RA $( \pm 1,0)$, marked by the light arrow, totally vanishes, which indicates that coupling between RA and LSPR is unrealizable when resonances are orthogonal to each other, and only parallel coupling is stimulated. However, LSPR with a broader bandwidth undergoes a dramatic redshift as $n_{i}$ increases. As the insulator index increases, the FWHM of SLR increases from $23\left(n_{i}=1.4\right)$ to $46 \mathrm{~nm}\left(n_{i}=1.8\right)$, and the reflectivity decreases from $25\left(n_{i}=1.4\right)$ to $9.3 \%\left(n_{i}=1.8\right)$, which indicates that insulators with higher indices can improve emissivity and slightly increase the FWHM. Typically, the FWHM of LSPR in the noble metal can be optimized down to $250 \mathrm{~nm}$ in the mid-infrared range, which is still inferior to the broadened SLR. Note that, in the broadband applications based on MIM structures, SLRs may experience unexpected cutoff at a broad working band, which degenerates their performance. However, a MIM structure with smaller $n_{i}$ suppresses the unexpected cutoff effectively and simultaneously reduces fabrication difficulty. Manufacture difficulty is reduced because larger disks are sufficient when a lower $n_{i}$ is adopted, thereby reducing the difficulty in fabricating smaller disks.

To better illustrate the above mechanisms, near-field distributions of normalized $|E|$ and $|\mathrm{Hx}|$ at the dips of $\operatorname{SLR}(0, \pm 1)$ and LSPR are indicated in Fig. 3. Electric dipole resonances were all stimulated inside disks with different strengths along the $y$-axis, as shown in Figs. 3(a)-3(c). However, LSPR has a greater strength electric field inside the insulator than SLR. Antisymmetric electric dipoles induced in disks and the mirror layer produce a magnetic dipole in the insulator [Fig. 3(d)], which can explain why LSPR is highly sensitive to the insulator index. For comparison, $|\mathrm{Hx}|$ in Fig. 3(e) is not only confined in the insulator layer, but also enhanced between the disks. Furthermore, this collective resonance dramatically increases when the insulator is replaced with a higher index layer, as indicated in Fig. 3(f). $|\mathrm{Hx}|$, among the disks, is caused by the coupling among LSPRs inside each disk through RA, which stimulates the collective SLR.

The corresponding MIM structures are fabricated on silicon substrates and patterned by ion beam etching after lithography technology. Insulators made of $\mathrm{SiO}_{2}$ or $\mathrm{SiNx}$ are prepared by

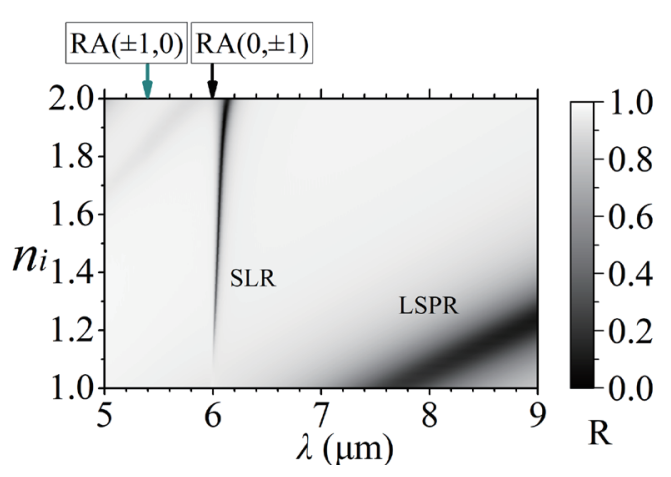

Fig. 2. (Color online) SLRs and LSPR transformation with increasing $n_{i}$. Corresponding geometrical parameters are as follows: $R=1.65 \mu \mathrm{m}, a_{x}=4.5 \mu \mathrm{m}, a_{y}=5 \mu \mathrm{m}, h_{m}=100 \mathrm{~nm}, h_{i}=180 \mathrm{~nm}, h_{d}=100 \mathrm{~nm}$, and $n_{e}=1.2$. Two fold, degenerated RA $( \pm 1,0)$ and RA $(0, \pm 1)$ propagating along the $x$ - and $y$-axes are indicated by arrows at 5.4 and 6 $\mu \mathrm{m}$, respectively. 


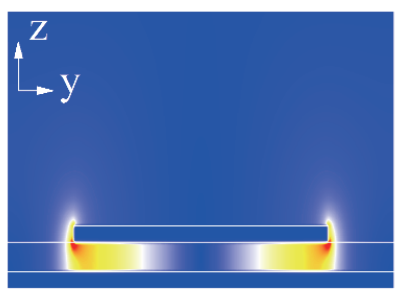

(a)

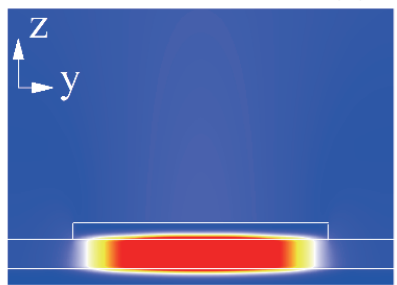

$\operatorname{LSPR}(n i=1.2)$

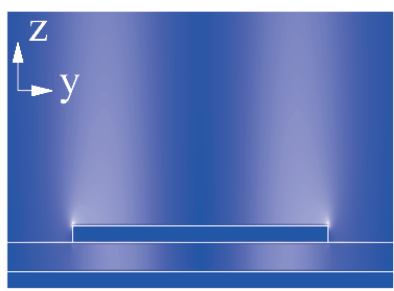

(b)

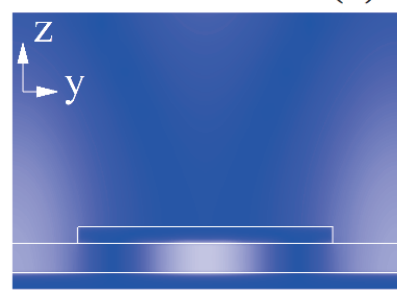

(d) $\operatorname{SLR}\left(n_{i}=1.2\right)$

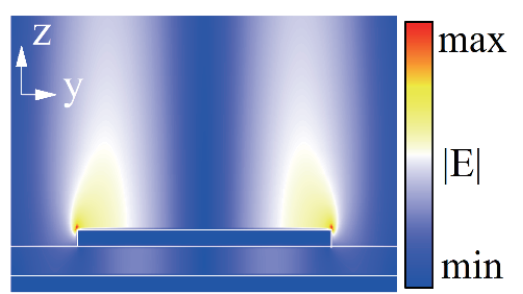

(c)

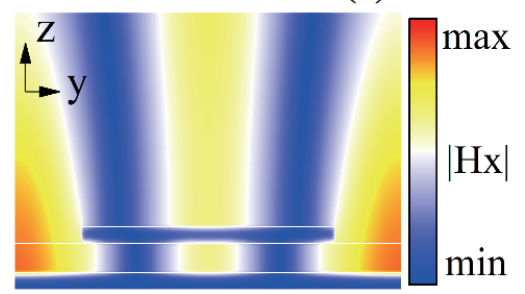

(f)

Fig. 3. (Color online) Normalized $|\mathrm{E}|$ and $|\mathrm{Hx}|$ field distributions at the dips of LSPR $\left(n_{i}=1.2\right)$ and SLR $\left(n_{i}=1.2\right.$ and 1.4), in which (a)-(c) and (d)-(f) share the same color bar as in (c) and (f), respectively.

plasma-enhanced chemical vapor deposition (PECVD) at $350{ }^{\circ} \mathrm{C}$, and the chamber pressures are 650 and 1000 mTorr, respectively. For $\mathrm{SiO}_{2}$, the synthetic gases are $\mathrm{SiH}_{4}(5 \mathrm{sccm})$ and $\mathrm{N}_{2} \mathrm{O}$ (32 sccm), and an RF power of $15 \mathrm{~W}$ was used, while for $\mathrm{SiNx}$, the synthetic gases are $\mathrm{SiH}_{4}(20$ $\mathrm{sccm})$ and $\mathrm{NH}_{3}(20 \mathrm{sccm})$, and an RF power of $20 \mathrm{~W}$ was used. Oblique and cross-sectional views of an individual metallic disk in an MIM structure are shown in SEM images in Fig. 1(b). Optical constants of dielectric films, $n$ and $k$, are indicated in Fig. 4(a), which were measured using an ellipsometer. In Fig. 4(a), the $k$ of insulators is equal to zero until the working wavelength exceeds $7.5 \mu \mathrm{m}$; however, the raised $k$ is still an order of magnitude less than that of metals. Losses of materials are the main reason for LSPR broadening; however, for SLR, radiation loss plays a major role.

Reflectance spectra are measured by the FTIR method under a TE-polarized oblique plane wave at $10^{\circ}$. In the experiments, grating constants along different axes are the same $\left(a_{x}=a_{y}\right.$ $=6.5 \mu \mathrm{m})$. However, under oblique incident light, overlapped RA $( \pm 1,0)$ splits into RA $(1,0)$ towards the longer wavelength and RA $(-1,0)$ towards the shorter wavelength (not shown). As shown in Fig. 4(b), overlapped SLR $(0, \pm 1)$ near RA $(0, \pm 1)$ is observed (indicated by an arrow). In addition, $\operatorname{SLR}(0, \pm 1)$ becomes stronger when the $\mathrm{SiO}_{2}$ insulator is replaced by $\mathrm{SiNx}$. From the optical constants in Fig. 4(a), the refractive indices of $\mathrm{SiO}_{2}$ and $\mathrm{SiNx}$ are respectively found to be 1.26 and 1.69 at RA $(0, \pm 1)$, which is consistent with the conclusion obtained in the simulations. However, SLRs in the experiments are much weaker and broader than those in the simulations. This discrepancy between the simulated and measured spectra might be attributed to the inconsistent radii of the disks, which degenerates the coupled efficiency among dipoles inside the disks and leads to a lower quality factor of SLRs in the experiments. Taking into account the imperfect fabrication as well as the measurement error, good agreement is still observed between the simulation and experimental results. 


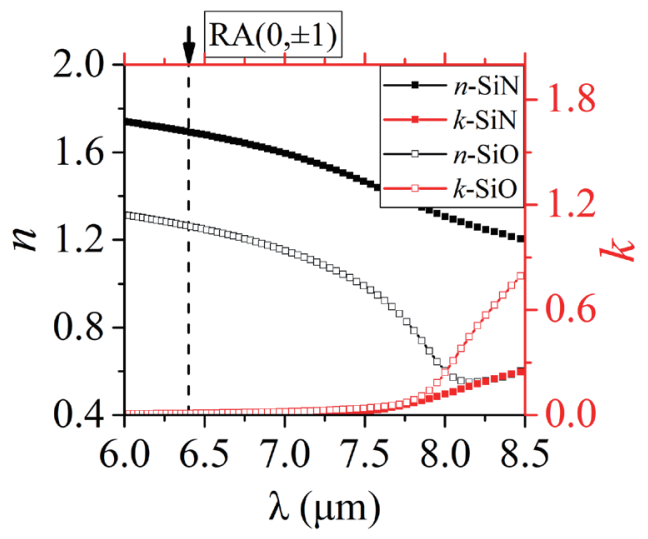

(a)

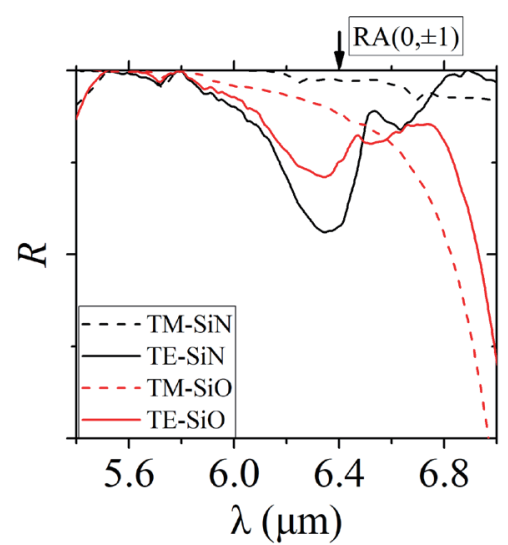

(b)

Fig. 4. (Color online) (a) Optical constants of insulators measured with ellipsometer (IR-VASE MARK II). The measured films were prepared by PECVD on silicon substrates of $200 \mathrm{~nm}$ and the fabrication conditions are identical to those of MIM structures. (b) Reflectance spectra of MIM structures with $\mathrm{SiO}_{2}$ or $\mathrm{SiNx}_{\text {insulators. }}$ Structure parameters in the sample are as follows: $R=1.6 \mu \mathrm{m}, a_{x}=a_{y}=6.5 \mu \mathrm{m}, h_{d}=h_{m}=100 \mathrm{~nm}$, and $h_{i}=180 \mathrm{~nm}$. Arrows indicate the positions of $\operatorname{RA}(0, \pm 1)$ in both figures at $\lambda=6.4 \mu \mathrm{m}$.

\section{Conclusions}

Ultra-narrowband SLRs based on parallel coupling in MIM structures were investigated by both simulation and experiment, and the parallel coupling was found to be enhanced easily by using a higher index insulator, even when homogenous medium conditions were not fulfilled. In the simulations, the FWHM and reflectivity of SLRs were as low as $46 \mathrm{~nm}$ and $9.3 \%$, respectively, when an insulator with $n_{i}=1.8$ was adopted. This work may further promote the usage of SLRs for high-performance NDIR gas sensors. Additionally, suppression effects were also verified in the simulations when an insulator with lower index was adopted, which can be used as reference in broadband applications to suppress the unexpected cutoff by SLRs in the working band.

\section{Acknowledgments}

We appreciate the financial support from the National Key Research and Development Program of China (Nos. 2017YFA0207103, 2017YFB0405403, and 2017YFA0206403), and the National Natural Science Foundation of China (No. 61475180).

\section{References}

1 J. J. Lai, H. F. Liang, Z. L. Peng, X. Yi, and X. F. Zhai: J. Phys. Conf. Ser. 276 (2011) 012129. https://doi. org/10.1088/1742-6596/276/1/012129

2 Y. Lee, S.-J. Kim, H. Park, and B. Lee: Sensors 17 (2017) 1726. https://doi.org/10.3390/s17081726

3 A. Lochbaum, Y. Fedoryshyn, A. Dorodnyy, U. Koch, C. Hafner, and J. Leuthold: ACS Photonics 4 (2017) 1371. https://doi.org/10.1021/acsphotonics.6b01025

4 N. I. Landy, S. Sajuyigbe, J. J. Mock, D. R. Smith, and W. J. Padilla: Phys. Rev. Lett. 100 (2008) 207402. https://doi.org/10.1103/PhysRevLett.100.207402 
5 I. Puscasu and W. L. Schaich: Appl. Phys. Lett 92 (2008) 233102. https://doi.org/10.1063/1.2938716

6 J. Hao, L. Zhou, and M. Qiu: Phys. Rev. B 83 (2011) 165107. https://doi.org/10.1103/PhysRevB.83.165107

7 J. Mertens, M.-E. Kleemann, R. Chikkaraddy, P. Narang, and J. J. Baumberg: Nano Lett. 17 (2017) 2568. https://doi.org/10.1021/acs.nanolett.7b00332

8 X. Liu, T. Tyler, T. Starr, A. F. Starr, N. M. Jokerst, and W. J. Padilla: Phys. Rev. Lett. 107 (2011) 045901. https://doi.org/10.1103/PhysRevLett.107.045901

9 P. Bouchon, C. Koechlin, F. Pardo, R. Haïdar, and J.-L. Pelouard: Opt. Lett. 37 (2012) 1038. https://doi. org/10.1364/OL.37.001038

10 S. Zou and G. C. Schatz: J. Chem. Phys. 121 (2004) 12606. https://doi.org/10.1063/1.1826036

11 E. M. Hicks, S. Zou, G. C. Schatz, K. G. Spears, R. P. Van Duyne, L. Gunnarsson, T. Rindzevicius, B. Kasemo, and M. Käll: Nano Lett. 5 (2005) 1065. https://doi.org/10.1021/n10505492

12 B. Auguié and W. L. Barnes: Phys. Rev. Lett. 101 (2008) 143902. https://doi.org/10.1103/ PhysRevLett.101.143902

13 Y. Chu, E. Schonbrun, T. Yang, and K. B. Crozier: Appl. Phys. Lett. 93 (2008) 181108. https://doi. org $/ 10.1063 / 1.3012365$

14 V. O. Byelobrov, T. L. Zinenko, K. Kobayashi, and A. I. Nosich: IEEE Antennas Propag. Mag. 57 (2015) 34. https://doi.org/10.1109/MAP.2015.2480083

15 P. P. Patra, R. Chikkaraddy, R. P. N. Tripathi, A. Dasgupta, and G. V. P. Kumar: Nat. Commun. 5 (2014) 1. https://doi.org/10.1038/ncomms5357

16 P. Offermans, M. C. Schaafsma, S. R. K. Rodriguez, Y. Zhang, M. Crego-Calama, S. H. Brongersma, and J. Gómez Rivas: ACS Nano 5 (2011) 5151. https://doi.org/10.1021/nn201227b

17 A. Abass, S. R.-K. Rodriguez, J. Gómez Rivas, and B. Maes: ACS Photonics 1 (2014) 61. https://doi. org $/ 10.1021 / \mathrm{ph} 400072 \mathrm{z}$

18 Y. Huang, L. Ma, M. Hou, and Z. Zhang: Plasmonics 11 (2016) 1377. https://doi.org/10.1007/s11468-016-01874

19 X. M. Bendaña and F. J. García de Abajo: Opt. Express 17 (2009) 18826. https://doi.org/10.1364/OE.17.018826

20 R. Adato, A. A. Yanik, C.-H. Wu, G. Shvets, and H. Altug: Opt. Express 18 (2010) 4526. https://doi. org/10.1364/OE.18.004526

21 A. G. Nikitin: Appl. Phys. Lett. 104 (2014) 061107. https://doi.org/10.1063/1.4864277

22 W. J. Wing, S. M. Sadeghi, and R. R. Gutha: J. Appl. Phys. 120 (2016) 234301. https://doi. org/10.1063/1.4972270

23 A. Vitrey, L. Aigouy, P. Prieto, J. M. García-Martín, and M. U. González: Nano Lett. 14 (2014) 2079. https:// doi.org/10.1021/n1500238h

24 L. Lin and Y. Yi: Opt. Express 23 (2015) 130. https://doi.org/10.1364/OE.23.000130

25 E. D. Palik: Handbook of Optical Constants of Solids (Academic Press, San Diego CA, 1998).

\section{About the Authors}

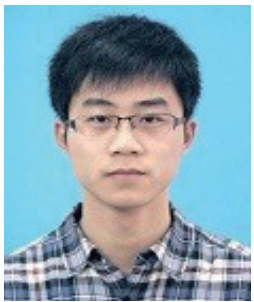

Aisheng Yu received his B.S. degree from East China Normal University in 2013. He is currently completing his doctorate degree at Science and Technology on Micro-System Laboratory, Shanghai Institute of Microsystem and Information Technology, Chinese Academy of Sciences. His research interests are in gas sensors and plasmonics.

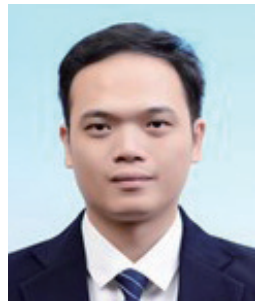

Wei Li received his Ph.D. double-degree in microelectronics and solid-state electronics from the Chinese Academy of Sciences, and in condensed matter and material physics from the University of Caen, France, in 2010. He is currently a professor at the State Key Laboratory of Functional Materials for Informatics. His research interests are in silicon-based micro-nanophotonic devices and physics, and optical/electromagnetic detection technology. 


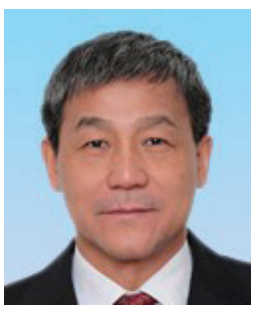

Yuelin Wang received his B.S. degree from Zhejiang University in 1982, his M.S. degree from the Harbin Institute of Technology in 1985, and his Ph.D. degree from Tsinghua University in 1989. He is currently a professor at the Shanghai Institute of Microsystem and Information Technology. His research interests are in micro-electromechanical and nano-electromechanical systems.

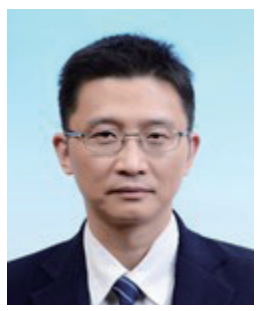

Tie Li received his B.S. and Ph.D. degrees in condensed matter physics from the University of Science and Technology of China, Hefei, in 1992 and 1997, respectively. He is currently a professor at the Science and Technology on Micro-System Laboratory at the Shanghai Institute of Microsystem and Information Technology. His research interests are in the mechanism, fabrication, and application of micro-nanofused sensors. (tli@mail.sim.ac.cn) 